\title{
Anabases
}

ANABASES Traditions et réceptions de l'Antiquité

$24 \mid 2016$

Varia

\section{L'inépuisable fécondité des anecdotes antiques sur la peinture. Présentation du site Pictor in Fabula}

Emmanuelle Hénin

\section{(2) OpenEdition}

Journals

Édition électronique

URL : http://journals.openedition.org/anabases/6014

DOI : 10.4000/anabases.6014

ISSN : 2256-9421

Éditeur

E.R.A.S.M.E.

Édition imprimée

Date de publication : 10 novembre 2016

Pagination : 297-310

ISSN : 1774-4296

\section{Référence électronique}

Emmanuelle Hénin, «L'inépuisable fécondité des anecdotes antiques sur la peinture. Présentation du site Pictor in Fabula », Anabases [En ligne], 24 | 2016, mis en ligne le 01 octobre 2019, consulté le 24 mars 2020. URL : http://journals.openedition.org/anabases/6014 ; DOI : https://doi.org/10.4000/anabases 6014

(c) Anabases 
Anabases 24 (2016), p. 297-310.

\section{L'inépuisable fécondité des anecdotes antiques sur la peinture. Présentation du site Pictor in Fabula}

Emmanuelle HÉNIN

L

e site Pictor in Fabula (www.pictorinfabula.com) est dédié à la fortune des “ traits exemplaires » relatifs à la peinture antique. L'expression « traits exemplaires » nous a semblé plus pertinente que le terme « anecdotes », qui connote quelque chose d'accessoire (“ anecdotique » est péjoratif), ou de purement descriptif ; le terme topos serait plus juste, mais on perd alors l'aspect concret. De plus, les passages retenus n'ont pas tous une forme narrative ; ils peuvent aussi être des jugements d'ordre général sur les peintres (du type : Polygnote peignait les hommes plus beaux ; Pauson les peignait plus laids ${ }^{1}$ ), voire sur la peinture (tel le paradoxe de la représentation chez Aristote $^{2}$ : on a du plaisir à voir les images même les objets terribles ou repoussants). Du point de vue rhétorique, les anecdotes ont le statut d'exemples au service de la démonstration. Et l'autorité d'un exemple ne vient pas de sa véracité (certains exemples sont fictifs, relevant alors de l'apologue ou de la fable), mais de sa récurrence, de sa réplicabilité : d'où la tendance des auteurs à répéter indéfiniment les mêmes anecdotes. Cependant, le mot exemple ou exemplum ne conviendrait pas non plus, car il est connoté moralement.

Dans l'Antiquité, les anecdotes sont un des lieux d'expression privilégié d'une théorie artistique qui est rarement formulée comme telle, mais souvent

1 * Piraicos, Dionysos et Pauson : portraits pires, semblables, meilleurs. Les titres des anecdotes présentes sur le site seront désormais mentionnées en petites capitales et précédées d'un astérisque ; on pourra retrouver sur le site toutes les références bibliographiques et iconographiques qui ne sont pas données ici par manque de place.

2 * Cadavres et bêtes sauvages, ou le PLAisir de la REPRÉSEnTATion. 
disséminée dans toute sorte de textes, ou formulée sur le mode de l'analogie : tel traité de philosophie, de logique ou d'éthique recourt à l'analogie picturale, et ce phénomène se poursuit en partie à l'époque moderne, malgré la spécificité des traités. En effet, l'idée même d'une histoire et d'une théorie de l'art vient des Grecs (c'est même une exception grecque, inconnue d'autres civilisations), et les principaux schémas en sont transposés de la Grèce à Rome, puis à la Renaissance : ainsi l'idée d'un progrès des arts, qui tend vers une représentation de plus en plus fidèle de la nature avant de connaître une période de décadence, vient de Xénocrate d'Athènes (sculpteur, début du III ${ }^{\mathrm{e}}$ siècle av. J.-C.), qui invente un vocabulaire artistique lié à la rhétorique. Xénocrate fixe un schéma de base, repris ensuite par Pline, puis par Alberti et Vasari, et enfin par Winckelmann ${ }^{3}$. Non seulement les grands concepts sont empruntés aux Grecs, mais leur formulation passe par la reprise des mêmes exemples.

Ces exemples sous-tendent toute la théorie artistique. Indéfiniment modulés, ils offrent un cadre contraignant à l'expression de la pensée de chaque théoricien, la contrainte rhétorique conditionnant la liberté théorique : ces anecdotes constituent un « ouvroir de théorie potentielle ». Par exemple, la théorie de la belle nature, qui domine la pensée esthétique $d u x^{e}$ au $x x^{e}$ siècle, se fonde sur l'Hélène de Zeuxis ${ }^{4}$, mais chaque manière de raconter l'anecdote est porteuse d'une interprétation nouvelle de ce concept. L'anecdote fonctionne comme une matrice théorique ; elle cristallise les enjeux de la théorie artistique et en éclaire les points aveugles. C'est pourquoi il est si éclairant de suivre le fil d'une anecdote depuis l'origine, afin de saisir la continuité d'une question esthétique ${ }^{5}$.

D'où l'idée de fournir au chercheur une base de données, pour lui éviter d'infinies recherches dans un corpus foisonnant. Pour l'Antiquité, le travail avait été commencé au $\mathrm{xvII}^{\mathrm{e}}$ siècle par Franciscus Junius, dont le traité, De Pictura veterum, collationne une quantité impressionnante de références à la peinture dans la littérature gréco-latine ${ }^{6}$. Il fut poursuivi par un érudit allemand, Johannes Adolph Overbeck, dont l'ouvrage Die antiken Schriftquellen zur Geschichte der bildenden Künste bei der Griechen (Leipzig, 1868) répertorie toutes les sources

3 Voir le colloque Histoire de l'histoire de l'art, t. I, De l'Antiquité au XVIII siècle, Paris, Klincksieck, "Conférences et colloques du Louvre », 1995, en particulier l'article de Salvatore Settis.

4 Zeuxis, Hélène.

5 * Sur l'Hélène de Zeuxis, on peut citer : F. Lecercle, La Chimère de Zeuxis, Tübingen, Gunter Narr, 1987 ; P. Sabbatino, La bellezza di Elena. L’imitazione nella letteratura e nelle arti figurative del Rinascimento, Florence, Olschki, 1997 ; ou encore E. C. Mansfield, Too Beautiful to Picture : Zeuxis, Myth, and Mimesis, Minneapolis, University of Minnesota Press, 2007.

6 Franciscus Junius, De pictura veterum, Amsterdam, J-G. Graevius, 1637 ; édition très augmentée, Rotterdam, R. Leers, 1694. 
antiques sur l'art en les classant par artiste et par ordre chronologique. Peu avant la première Guerre mondiale, Adolphe Reinach entreprit de traduire ce recueil en français, tout en le complétant par une abondante documentation archéologique pour le rendre accessible à un public plus large. En partant pour le front le 1er août 1914, il confia à son oncle la tâche de publier la partie de ses manuscrits portant sur la peinture, pour que son “ énorme labeur » ne fût pas perdu ; « ainsi, même si je disparaissais, le premier volume de notre Recueil pourrait paraître ». Il mourut dans les Ardennes le 30 août, à 27 ans. Son recueil a été édité par son oncle et réédité par Agnès Rouveret en 1985 ; quant aux textes d'Overbeck sur la sculpture, ils ont été traduits seulement en $2002^{7}$. En 2014, un groupe de chercheurs allemands a édité une version considérablement augmentée et remaniée du recueil d'Overbeck, en cinq volumes, intégrant quantité de textes nouveaux et des commentaires tenant compte des dernières découvertes archéologiques ${ }^{8}$. Cette nouvelle édition rétablit les passages arbitrairement coupés par Reinach, mais sa vocation est de constituer un recueil de documents sur des œuvres perdues ; dans la perspective de la base PIF, où les topoi constituent un réservoir d'arguments, il importe de rétablir le contexte de ces extraits, qui montrent la plasticité des lieux communs empruntés à l'art. Ainsi, dans le De divinatione, Cicéron parle de la Vénus d'Apelle à propos du hasard ${ }^{9}$; Horace évoque les rapports d'Alexandre et d'Apelle non pour faire l'éloge du souverain mécène, mais pour lui reprocher au contraire de ne rien y connaître en littérature ${ }^{10}$; Clément d'Alexandrie blâme les courtisanes en donnant l'exemple de Phryné, modèle d'Apelle et de Praxitèle" ; Plutarque cite l'anecdote de Zeuxis et Agatharcos à propos de l'importance de se faire des amis qui durent longtemps ${ }^{12}$.

Si les sources antiques ont été balisées de longue date, les sources modernes n'avaient jamais fait l'objet d'un repérage, tant la tâche semble illimitée. En effet, comme dans l'Antiquité, la théorie artistique est disséminée dans toutes sortes de sources : outre la littérature artistique proprement dite (traités d'art, vies d'artistes, guides touristiques, comptes rendus d'expositions), elle est présente sous forme d'analogie dans de nombreux discours théoriques : poétiques et traités de rhétorique, traités de morale et de théologie, traités de philosophie et manuels

7 A. Reinach, La Peinture ancienne. Textes grecs et latins, introduction et notes par A. Rouveret, Paris, Macula, 1985 ; La Sculpture grecque. Sources littéraires et épigraphiques, trad. M. Muller-Dufeu, Paris, ENSBA, 2002.

8 Der Neue Overbeck. Die antiken Schriftquellen zu den bildenden Künsten der Griechen, éd. S. Kansteiner, K. Hallof, L. Lehmann, B. Seidensticker, K. Stemmer, Berlin, De Gruyter, 2014.

9 * Apelle, Vénus anadyomène.

10 * Apelle et Alexandre.

11 * Apelle, Praxitele et Phryné.

$12 *$ ZeuXis et Agatharcos. 
de civilité. Il n'est pas de champ d'où elle soit exclue : relations de fêtes et de voyages, préfaces, correspondances, romans, nouvelles et tout le champ de la fiction.

La liste des soixante-dix topoi retenus pour ce corpus numérique s'est dessinée peu à peu, après plusieurs années de recherche. Plusieurs principes ont présidé à ce choix :

- la fécondité des anecdotes antiques pour la théorie artistique de l'époque moderne ( $\mathrm{xv}^{\mathrm{e}}-\mathrm{xvIII}{ }^{\mathrm{e}}$ siècle). Ainsi, certains tableaux d'Apelle ont été retenus et pas d'autres : Alexandre au foudre, parce qu'il a le bras en raccourci et qu'il donne le modèle du portrait du souverain en majesté (Félibien en fait un modèle de portrait royal) ${ }^{13}$; ou la Diane parce qu'elle est un paradigme de l'ut pictura poesis, Apelle ayant dépassé Homère ${ }^{14}$; mais pas, par exemple, la Famille de Centaures ou l'Hercule terrassant les serpents dont il n'est rien dit de particulier.

- ont été écartées (faute de temps) la plupart des anecdotes sur la sculpture, sauf celles qui revenaient fréquemment dans les traités de peinture, soit qu'elles mettent en parallèle peinture et sculpture, soit qu'elles servent à conceptualiser des questions de théorie picturale : la Vénus de Praxitèle est très souvent associée à la Vénus d'Apelle, tout en illustrant les rapports entre amour et création artistique $^{15}$; le Zeus de Phidias constitue depuis Cicéron l'équivalent de l'Hélène de Zeuxis dans la réflexion sur la représentation idéale ${ }^{16}$; la Vache de Myron est le pendant sculptural des raisins de Zeuxis, et fait l'objet d'une grande fortune épigrammatique ${ }^{17}$.

- enfin, les anecdotes modernes n'ont été retenues que dans deux cas : quand elles sont explicitement reliées à une anecdote antique (ainsi le concours de la ligne entre Zeuxis et Parrhasios, souvent rapproché de l'O de Giotto ${ }^{18}$ ), ou du moins quand la transposition est évidente. Mosini raconte ainsi comment Annibal Carrache, pour se venger d'un commanditaire qui le prenait de haut, peignit un rideau sur un miroir, et se moqua du commanditaire quand il voulut soulever le rideau : transposition évidente de la victoire de Parrhasios sur Zeuxis ${ }^{19}$.

\section{Les enjeux théoriques de ces " traits exemplaires »}

Les anecdotes renvoient aux grandes questions de la théorie picturale et sont souvent redondantes entre elles, plusieurs pouvant démontrer la même

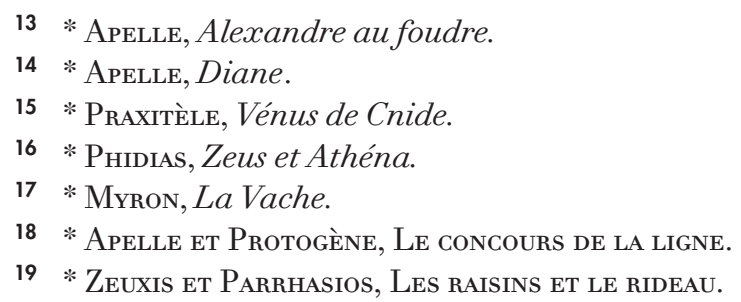


idée. Inversement, certaines se relient à plusieurs enjeux à la fois, et ces enjeux englobent tout le circuit de l'œuvre d'art : du statut social de l'artiste à la manière dont il crée, jusqu’à la réception de ses œuvres par le public.

1. LA Dignité DE L'ART est illustrée par une série d'anecdotes. À partir de la Renaissance, la revendication des peintres de voir la peinture accéder au rang d'art libéral s'appuie sur le précédent de Pamphile, le maitre d'Apelle ${ }^{20}$ : modèle du peintre humaniste, érudit dans les belles lettres et la géométrie, mais aussi bon enseignant, puisqu'il avait fondé une école ; et bien payé, puisqu'il demandait une grosse somme à ses élèves (un talent par an), somme que chaque théoricien transpose dans sa monnaie pour prouver le prestige de la peinture - tout comme les prix des tableaux antiques, souvent rappelés par Pline. Autre aspect important pour la sociologie des peintres : Pline consacre un chapitre aux femmes peintres, et ce chapitre connut une fortune importante grâce à Boccace et à ses illustrations, avant d'être cité pour légitimer le talent des femmes peintres modernes, de Lavinia Fontana à Rosalba Carriera ${ }^{21}$.

Autres épisodes célèbres : Démétrios renonçant à détruire Rhodes pour épargner l'Ialysos de Protogène ${ }^{22}$; Alexandre donnant à Apelle sa maîtresse Campaspe, sujet souvent illustré par les peintres ${ }^{23}$. Ces anecdotes sont reprises pour justifier la place de l'artiste dans la société, et assorties d'équivalents modernes, montrant les peintres parlant d'égal à égal avec les souverains, tel Charles Quint ramassant le pinceau de Titien, ou Vélasquez admis dans l'intimité de Philippe IV. L'anecdote d'Apelle et Campaspe est la seule à faire l'objet d'une fortune particulière au théâtre : outre la Campaspe de John Lily (1584), une comédie de Calderon, Dar todo y dar nada (1651), puis en France, une série de ballets et d'opéras au tournant des $\mathrm{XVIII}^{\mathrm{e}}$ et $\mathrm{xIX}^{\mathrm{e}}$ siècles. La fortune de l'épisode est telle que Noverre le choisit pour son ballet-pantomime dansé à Vienne en 1774, puis à Paris en 1776, parce qu'il permet aux spectateurs de suivre une action entièrement muette : la danse assume pleinement le statut d'art mimétique, prouve sa capacité à représenter une action à l'égal de la tragédie ou de la peinture ; d'où le choix d'Apelle, pour souligner le parallèle de la peinture et de la danse. La dignité de la peinture plaide pour la dignité de la danse, à une époque où elle lutte à son tour pour faire reconnaitre sa valeur sociale.

2. L'ethos de L'ARTiste occupe une place importante. En particulier, l'orgueil ou la modestie des artistes apparaissent dans de nombreuses anecdotes sur leur rapport à l'argent et à la renommée. Chez Pline, on a l'impression que les

\footnotetext{
20 * Pamphile et la peinture comme art libÉRAL.

21 * Femmes peintres.

22 * Рrotogène, Ialysos.

23 * Apelle et Campaspe.
} 
tempéraments font système, comme dans une histoire moralisée de la peinture : à l'orgueil de Parrhasios et de Zeuxis s'oppose l'humilité d'Apelle et de Protogène ${ }^{24}$. En effet, Zeuxis portait un manteau brodé d'or avec son nom, faisait payer pour voir ses tableaux, puis au contraire les offrait en disant qu'aucun prix n'était digne d'eux ${ }^{25}$. À l'inverse, Apelle incarne la modestie : il reconnaît la supériorité de ses rivaux sur des points précis ; il est attentif à la critique ; il signe ses tableaux à l'imparfait, “Apelles faciebat », pour montrer qu'ils sont toujours perfectibles, faisant montre d'une modestie plus que suspecte, puisque chez les peintres à partir du Xve siècle et jusqu'à David, la signature à l'imparfait est plutôt un moyen de se distinguer en se réclamant d'un exemple prestigieux ${ }^{26}$. Plus modeste encore, Protogène se contente d'une modeste chaumière et se nourrit exclusivement de lupins bouillis ${ }^{27}$. Son ascétisme confine à la mélancolie. L'anorexie des peintres est souvent reprise à l'époque moderne - ainsi à propos de Piero di Cosimo, ou de Michel-Ange, qui ne prit que du pain et du vin tant qu'il peignait le Jugement dernier. Quant à Parrhasios, il cultive une prédilection pour les sujets morbides, tels Philoctète ou Prométhée torturé sur son rocher ${ }^{28}$. Il y a donc dès la Grèce ancienne une idiosyncrasie du génie, et Vasari n’a fait qu'accentuer la tendance, déjà présente chez Pline et ses sources, à relier les progrès de l'art à une série d'individus exceptionnels.

3. Le jugement du public. C'est l'enjeu de l'anecdote bien connue d'Apelle et $\mathrm{du}$ cordonnier ${ }^{29}$. Quand il avait fini un tableau, Apelle se cachait derrière pour recueillir le jugement du public ; un cordonnier lui fit remarquer un défaut à une chaussure, que l'artiste corrigea ; mais quand le lendemain il s'avisa de critiquer aussi la jambe, Apelle répondit: «ne sutorultra crepidam ». Autrement dit, le peintre fait confiance au jugement du public, mais il fait valoir sa compétence spécifique, en l'occurrence dans le rendu de l'anatomie. L'anecdote est source d'anecdotes parallèles dès l'Antiquité : Lucien raconte comment Phidias se cacha derrière son Zeus et le modifia en fonction des réactions du public. Mais aussi d'anecdotes antithétiques : Polyclète, selon Élien, fit deux sculptures, l'une en suivant les avis du public, l'autre selon son propre instinct, et le peuple jugea la seconde beaucoup plus réussie. La question est très débattue en France au xvıII ${ }^{\mathrm{e}}$ siècle, quand les Salons s'ouvrent au grand public: qui est capable d'émettre un jugement de goût? le peintre, les critiques, les honnêtes gens, ou tout visiteur du Salon ?

\footnotetext{
$24 *$ Parrhasios : OrgueIL.

$25 *$ ZeUXIs ET LA RICHeSSE.

26 * ApELles faCiebat : LA Signature À L'IMPARFAit.

27 * РвотоgÈne, Ialysos.

28 * Parrhasios, Philoctète; Parrhasios, Prométhée.

29 * Apelle et le cordonNier.
} 
Dès l'instant où tout le monde se pique de juger la peinture, les artistes se sentent destitués de leurs prérogatives.

4. La postérité. Plusieurs anecdotes témoignent que les artistes antiques avaient le souci de la postérité : Phidias avait inclus son portrait au centre du bouclier de sa Minerve; Sostrate de Cnide avait recouvert sa signature, sur le phare d'Alexandrie, d'une couche de ciment qui dévoila son nom à la postérité. De même, Protogène avait mis quatre couches de peinture sur son tableau de Ialysos ${ }^{30}$, pour que le temps effaçant l'une, découvre la suivante. Bien entendu, cette anecdote est fortement mise en doute par les modernes, qui soulignent l'impossibilité d'une telle performance. Même les plus ardents défenseurs des Anciens reconnaissent qu'on ne peut prendre Pline à la lettre : dès 1667, Dati propose de comprendre que Protogène est revenu à quatre reprises sur son tableau pour l'améliorer. Durand (1725), suivi par Caylus, comprend que le tableau est « bien empâté ».

5. L'autonomie de l'art. C'est une des lectures possibles de l'anecdote des premiers peintres, qui écrivaient sur leur tableau “ ceci est un bœuf » : elle permet de critiquer l'insuffisance du langage pictural, qui a besoin des béquilles des mots et donc de signes artificiels. Cette lecture est très nette dans toutes les histoires “ téléologiques » de la peinture, qui mettent en regard la maladresse des primitifs et les progrès accomplis depuis ${ }^{31}$. Vasari se moque ainsi des peintres du Quattrocento qui ont recours à l'écriture, tel Orcagna dans le Triomphe de la Mort du Camposanto (1340). Cette fresque, aujourd'hui attribuée à Buffalmaco et très abîmée, contenait de nombreuses inscriptions poétiques, placées tantôt dans des phylactères, dans la bouche des vieillards, tantôt dans des cartouches en marge de l'image ${ }^{32}$.

6. L'illusionnisme. De nombreuses anecdotes accréditent l'idée que le sommet de l'art est l'illusion du réel. Les raisins de Zeuxis ${ }^{33}$, le cheval d'Apelle ${ }^{34}$, les Hoplites de Parrhasios ${ }^{35}$, les tuiles du théâtre de Claudius Pulcher où les oiseaux viennent se poser ${ }^{36}$, le Satyre de Protogène ${ }^{37}$, la Vache de Myron ${ }^{38}$. À l'époque moderne, elles sont transposées : le portrait de Paul III mis par Titien à sécher sur

\footnotetext{
30 * Protogìne, Ialysos.

31 * Peintres archaïques.

32 La fortune de l'anecdote est telle que j'ai pu lui consacrer un livre, Ceci est un boeuf: la querelle des inscriptions dans la peinture, Turnhout, Brepols, 2013.

33 * ZeuXis et Parrhasios, les raisins et le Rideau.

$34 *$ Apelle, Le Cheval.

35 * Parrhasios, Les Hoplites.

36 * Les oiseaux picorent les tuiles du théâtre de Claudius Pulcher.

37 * Рвотоgène, Satyre et parergia.

38 * Myron, La Vache.
} 
une fenêtre, était salué par les passants (selon Varchi), tout comme la Jeune fille à la fenêtre de Rembrandt (1645). Cette idée s'enracine dans le topos antique des vultus viventes. Mais ces anecdotes ne sont pas univoques, car à l'époque hellénistique, la mimesis se voit supplantée par la phantasia, de sorte que l'anecdote des raisins de Zeuxis est doublée d'un épilogue : Zeuxis avait peint un jeune garçon portant une corbeille de raisins (thème repris par Caravage et Hans von Aachen), que les oiseaux vinrent becqueter ${ }^{39}$. Furieux, Zeuxis effaça les raisins et y vit une preuve de son échec, car il avait mieux peint les raisins que le jeune garçon, faute de quoi les oiseaux auraient eu peur de lui. La même aventure arriva au satyre et à la perdrix de Protogène, selon Strabon : les passants ne s'extasiaient que sur la perdrix, de sorte que Protogène l'effaça, car elle était accessoire à ses yeux ${ }^{40}$.

7. La belle nature. Ce thème est incarné par l'anecdote de Zeuxis et les cinq jeunes filles de Crotone, au fondement de l'idéalisme qui régit l'esthétique moderne : l'artiste doit choisir et combiner les beautés de la nature ${ }^{41}$. Cependant, les interprétations diffèrent : l'artiste part-il d'une idée de la beauté (Platon, Cicéron), ou bien combine-t-il des éléments de l'expérience (Aristote)? L'anecdote cristallise une autre critique : ce portrait en patchwork est incompatible avec la définition de la beauté comme unité et harmonie, critique formulée dès la Renaissance et reprise par Francis Bacon (OfBeauty).

8. LE TEMPs de LA CRÉAtion. Plusieurs anecdotes révèlent une tension entre le goût du geste prompt et virtuose, et la reconnaissance de la lenteur du processus créateur. Critiquant son collègue Agatharcos, Zeuxis oppose la rapidité et la facilité de l'exécution au " travail approfondi qui fait durer une œuvre » et au "souci d'exactitude que comporte la beauté parfaite »(Plutarque) ${ }^{42}$. Selon le peintre, le temps employé à l'exécution d'une peinture est directement lié à sa bonne conservation. Pour la même raison, Protogène passait quatre couches de peinture sur ses tableaux pour en assurer la pérennité. Cependant, à force d'application, Protogène ne mit pas moins de sept ans à achever son Ialysos. C'est pourquoi Apelle lui reproche sa « nimia diligentia », son excès de soin, thème appelé à une grande fortune à la Renaissance, tant dans les traités d'art que dans les emblèmes (Guillaume de La Perrière, Pierre Cousteau) ${ }^{\mathbf{4 3}}$.

9. La hiÉRARChiE DEs GenRes. Pline consacre un développement aux peintres qui se sont illustrés dans des « minores picturae ». Il ne définit pas précisément en quoi consistent ces « plus petites peintures », mais il remarque, à propos de Piraicus -

\footnotetext{
39 * Zeuxis et Parrhasios, les raisins et le Rideau.

40 * Рвотоgène, Satyre et parergia.

$41 *$ Zeuxis, Hélène.

$42 *$ Zeuxis et Agatharcos.

43 * Apelle et la nimia diligentia.
} 
qualifié de « rhyparographe » (peintre d'objets vils) -, qu'il s'est spécialisé dans le traitement de sujets bas (humilia) : « des boutiques de barbiers et de cordonniers, des ânes, des comestibles et d'autres sujets du même ordre »"4 ${ }^{44}$ Au XVII siècle, les théoriciens hollandais (de Van Mander à Hoogstraten) revendiquent l'exemple de Piraicus et des autres peintres spécialisés pour justifier les genres spécifiques : Ludius justifie la peinture de paysage ${ }^{45}$; le Boeuf de Pausias, la peinture animalière ${ }^{46}$; et la Glycère du même Pausias, la peinture de fleurs ${ }^{47}$. Parallèlement, les poétiques et traités d'art ont tendance à transposer à la peinture une hiérarchie valable pour le théâtre (tragédie/comédie) et pour la rhétorique (style élevé/moyen/bas). Au xvis ${ }^{e}$ siècle, le chapitre de Pline sur la minor pictura et les analogies d'Aristote entre peintres et dramaturges sont ainsi utilisées ensemble dans le débat sur la hiérarchie des genres : le credo académique (incarné par Bellori) s'appuie sur Aristote pour condamner les peintres comme Caravage ou les Bamboccianti, qui traitent des mêmes objets que les rhyparographes antiques, Pauson et Pireicus ${ }^{48}$. À l'inverse, Pireicus est donné en modèle pour justifier le statut de la peinture de genre en Espagne, où il est comparé à Vélasquez.

10. Les Questions DE TEChNiQue PICtURALE sont au centre de plusieurs anecdotes, surtout chez Pline ${ }^{49}$. Particulièrement corrompu, le passage décrivant les effets de l'atramentum d'Apelle, invention tenue secrète par le peintre, fait l'objet des interprétations les plus variées ${ }^{50}$. Selon Pline, il consistait à passer une couche de pigment sombre (ou de vernis) à la surface des tableaux pour les protéger tout en empêchant que “ l'éclat des couleurs ne blessât la vue » en donnant de l'austérité (austeritas) aux couleurs trop éclatantes - mais comment ? En revanche, les observations de Pline sur le relief du Boeuf de Pausias et de l'Alexandre au foudre d'Apelle semblent plus faciles à comprendre et à transposer dans le langage de la peinture moderne ${ }^{51}$. Pausias aurait inventé un procédé pour suggérer l'illusion du volume, que les commentateurs identifient au raccourci, tout en le reliant volontiers au clair-obscur. Quant à l'Alexandre au foudre dont « les doigts semblent

\footnotetext{
44 * Piraicos et la rhyparographie.

$45 *$ Ludius peintre de paysages.

$46 *$ Pausias, Le Boeuf.

47 * Pausias et la bouQuetière Glycère.

48 * Piraicos et la rhyparographie ; Piraicos, Dionysos et Pauson : portraits pires, semBLABLES, MeILleurs.

49 Ces questions sont minutieusement examinées par M. BERT, Lectures, réécritures et peintures à partir de Pline l'Ancien. La réception de l'Histoire naturelle en Italie, de Pétrarque à Vasari, thèse de doctorat, Université de Liège/Université de Paris I-Sorbonne-Panthéon, 3 vol., 2011-2012.

$50 *$ ApElle, atramentum.

51 * Pausias, Le Boeuf; Apelle, Alexandre au foudre.
} 
en relief et le foudre sortir du tableau ", il est mentionné dès Ghiberti et suscite des commentaires sur le raccourci, le clair-obscur, l'éclairage et le traitement des carnations. En outre, Apelle avait réalisé cette œuvre à l'aide de seulement quatre couleurs. Pline affirme ailleurs (xxxv, 50), de même que Cicéron (Brutus, 70), le fait que les meilleurs artistes se contentaient de quatre couleurs. Cependant, ce point est peu repris à la Renaissance : seul Érasme loue Dürer d'avoir surpassé Apelle en n'utilisant que deux couleurs. C'est seulement au xvıI ${ }^{\mathrm{e}}$ siècle que cette économie de moyens trouve sa place dans le fantasme de la peinture antique, et fait l'objet d'abondants commentaires.

11. Le dessin et la couleur. Dans le débat sur le dessin et la couleur, les anecdotes illusionnistes sont régulièrement reprises en faveur de la couleur, notamment chez Pino et Dolce qui défendent la peinture vénitienne. À l'inverse, les partisans du dessin invoquent le concours de la ligne la plus fine (linea summae tenuitatis) entre Apelle et Protogène ${ }^{52}$. Il s'agit de l'anecdote la plus controversée, entre le $\mathrm{xv}^{\mathrm{e}}$ et le $\mathrm{xIx}^{\mathrm{e}}$ siècle, tant son sens littéral est obscur : Apelle va rendre visite à Protogène et, ne le trouvant pas chez lui, il dessine une ligne très fine sur une toile blanche pour signer son passage. Protogène revient, trouve la ligne, comprend qu'Apelle est passé, et " fend » la ligne d'une autre plus fine; Apelle revient et en fait autant. Selon Pline, ce tableau, qui ne présentait que des lignes presque invisibles, fut transporté dans le palais d'Auguste où il jouissait d'une immense admiration. D'où des débats sans fin : en quoi consistaient ces lignes? chacun avait-il tracé une ligne dans l'épaisseur de la précédente? Ou bien étaient-ce les contours de plusieurs figures qui s'entrelaçaient, comme le pensent la plupart des théoriciens (Van Mander, De Piles, etc.) ? Était-ce un exercice de perspective (Ghiberti), ou une étude de nu (Quatremère) ? L'anecdote est réinterprétée en fonction des intérêts de chaque théoricien. Mais ses implications paraissent choquantes : qu'un tableau abstrait puisse être admiré pendant des années, dans le palais d'Auguste ; que l'habileté technique domine le souci de la figuration, et que le dessin soit ainsi présenté comme le tout de la peinture. Selon Cochin, qui annote en 1760 l'article de Germain, “ Cette histoire n'est intelligible qu'en supposant que ces traits représentassent quelque chose, comme seroit une tête de profil ». De même, l'anecdote de la jeune fille de Corinthe connaît une grande fortune dans la peinture néoclassique (Wright of Derby, Suvée, Girodet, Allan), correspondant à un nouvel assaut des partisans du dessin ${ }^{53}$. Ici encore, la lettre de l'histoire n'est pas très claire (s'applique-t-elle à la peinture, à la sculpture ou aux deux à la fois ?); mais faire de l'ombre l'origine de la peinture revient en tout cas à exalter la ligne et le contour, comme le font les contemporains de David et Ingres.

\footnotetext{
52 * Apelle et Protogène : le concours de la ligne.

53 * Dibutade et la Jeune fille de Corinthe.
} 
12. L'expression et la communication des passions. Cet enjeu est central dans la théorie artistique, et ce depuis l'Antiquité. L'expression des passions est considérée comme un talent nécessaire au peintre, comme le montre un dialogue des Mémorables où Socrate rend visite à Parrhasios et l'interroge sur le véritable objet de son art ; ce dialogue est particulièrement glosé dans les traités anglais ${ }^{54}$. Encore faut-il distinguer deux types de passions, qu'on pourrait dire “ éthiques » et “ pathétiques », selon une distinction de Quintilien appliquée à la peinture par Roger de Piles ${ }^{55}$. Aristote affirme préférer Polygnote, peintre de la vieille école, qui donne la priorité aux caractères sur les passions, à Zeuxis qui fait l'inverse ${ }^{56}$. Aristote juge comme une décadence l'évolution de la peinture hellénistique vers plus de pathétique, évolution dont témoignent plusieurs exemples : Aristide de Thèbes suscite la pitié en peignant une Mère mourante dont l'enfant suce du sang au lieu de lait ${ }^{57}$; Timomaque crée la terreur en représentant dans sa Médée le dilemme entre colère et amour maternel ${ }^{58}$; Parrhasios parvient à combiner une dizaine de passions dans son allégorie du Peuple athénien, exploit qui paraît très suspect aux modernes ${ }^{59}$. Timanthe représente toute la gamme des passions tristes parmi les assistants au Sacrifice d'Iphigénie, et voile le visage d'Agamemnon pour exprimer le comble de la douleur ${ }^{60}$. L'anecdote, parmi les plus commentées, porte sur les limites de l'expression et rejoint au xvıII ${ }^{\mathrm{e}}$ siècle la réflexion de Lessing dans le Laocoon. D'autant que le sujet étant commun à la tragédie et à la peinture, il permet lui aussi de penser la spécificité des deux arts. À l'instar des théoriciens, les peintres prennent parti pour ou contre le voile de Timanthe : Tiepolo voile le visage d'Agamemnon, fidèle à l'anecdote antique, mais Van Loo se targue de le montrer, affichant la supériorité des modernes sur les anciens en matière d'expression.

Les passions pathétiques se déploient particulièrement dans les sujets violents ou macabres, comme la mère mourante d'Aristide ; mais ce n'est pas sans retenue, ni respect d'un certain décorum. On loue ainsi Timomaque de n'avoir pas peint le moment où Médée tue ses enfants : le peintre a choisi l'instant précédent le crime, à la fois plus décent et plus efficace sur le spectateur, comme le fait encore Delacroix $^{61}$. La peinture de Timomaque est l'objet, dès l'Antiquité, d'une réflexion sur

\footnotetext{
54 * Parrhasios et Socrate : le dialogue sur les passions.

55 Quintilien, VI, 2 ; Roger de Piles, Cours de peinture par principes, 1708, éd. 1989, Gallimard, TEL, p. 92.

56 * Zeuxis et Polygnote : action et caractères.

57 * Aristide de Thèbes : la mère mourante, le malade.

58 * Timomaque, Ajax et Médée.

59 * Parrhasios, Le Peuple d'Athènes.

60 * Timanthe, Iphigénie.

61 * Timomaque, Médée.
} 
l'instant fécond qui anticipe celle de Lessing. Les sujets macabres, comme Jocaste ou Philoctète, suscitent-ils l'horreur, ou au contraire produisent-ils une sorte de catharsis picturale? La question est très débattue au XvıII ${ }^{e}$ siècle, en lien avec le goût des spectacles pathétiques. Mais plus profondément, elle pose la question des limites ultimes de l'art : il est dit qu'Apelle peignait non seulement des mourants, mais des orages, le tonnerre, la foudre et les éclairs, « et même ce qui ne peut pas être peint (et quae pingi non possunt) » : défi que relève Poussin dans son Paysage à l'orage, à en croire son biographe Félibien ${ }^{62}$.

13. Le DANGER DU BRoUillage DES Frontières ENTRE LE RÉEL ET SA REPRÉSENTATION. De nombreuses anecdotes illustrent ce danger, telle l'histoire de l'acteur Polus qui, pour jouer le rôle d'Électre croyant Oreste mort, mit dans l'urne les cendres de son propre fils qui venait de mourir. Elle illustre la nécessité d'être sincèrement ému pour émouvoir, et l'adage horatien : si vis me flere, dolendum est primum tibi. En quelque sorte, cette anecdote sert d'illustration à un topos rhétorique, tous deux étant cités ensemble dans les traités de poétique et de peinture. À l'Âge classique prévaut l'interprétation empathique de ce précepte, fondée sur une lecture littérale de l'Ion de Platon; tandis que le xviıI siècle, avec le paradoxe du comédien, redécouvre une attitude de distanciation déjà exprimée par les Stoïciens. Une autre anecdote montre le danger de vouloir conformer le modèle à la copie : celle de Parrhasios torturant à mort un esclave olynthien pour peindre les souffrances de Prométhée, reprise à propos d'un Christ en croix de MichelAnge : cette fois, le décalque est manifeste, et se trouve pour la première fois sous la plume d'Andreas Schott, qui la tire d'une histoire circulant dans les couloirs du Collegio Romano ${ }^{63}$. Cette utopie d'abolir les frontières de la fiction rejoint des théories contemporaines du jeu théâtral ou de la performance artistique.

Comme on voit, ces anecdotes sont loin d'être limpides : soit que leur lecture littérale pose problème, soit qu'elles interrogent l'impossible de la représentation : l'abstraction, l'extrême violence, l'abolition de la fiction dans la réalité. En outre, l'attitude envers ces lieux communs évolue selon les époques, d'où l'importance de les envisager dans une perspective diachronique. La première Renaissance nourrit une grande admiration pour l'Antiquité et cherche à s'approprier le prestige des tableaux perdus en les réinventant par l'imitation : Botticelli se montre un nouvel Apelle en recréant la Vénus anadyomène, considérée comme le chefd'œuvre du peintre, suivi par Titien, encore plus proche du texte de Pline. À partir de 1550, quand les traités d'art se multiplient, leurs auteurs recherchent des parallèles entre peintres antiques et peintres modernes : par exemple, le concours

62 * Apelle et l'irreprésentable.

63 Andreas Sснотт, M. Annaei Senecae Rhetoris Suasoriae Controversiae Declamationumque Excerpta, Heidelberg, Jérôme Commelin, 1604, p. 219. * Parrhasios, Prométhée. 
de la ligne la plus fine entre Apelle et Protogène est rapproché de l'O de Giotto. Les théoriciens manient volontiers le raisonnement analogique, en transposant les exemples d'un domaine à l'autre, de la peinture à la morale, à la théologie ou à la spiritualité - tel François de Sales. Tout change au xvII siècle avec la querelle des Anciens et des Modernes : l'autorité de Pline est violemment remise en cause, et les anecdotes traitées de fables et d'historiettes naïves, sans fondement historique ni vraisemblance. Ce courant prend naissance en Italie dans les années 1620, avec les pamphlets d'Alessandro Tassoni (1620) et de l'abbé Lancelloti (1623 et 1636), qui taxent respectivement de « romans grecs » et « d'inepties solennelles » les anecdotes pliniennes, et dénigrent les exploits des peintres antiques en les confrontant systématiquement aux peintres modernes ${ }^{64}$. Ce sont ces textes peu connus qui inspirent aux Félibien et Perrault, courtisans de Louis XIV, leurs sarcasmes envers la peinture antique, au service de l'idéologie monarchique et nationale. Ces critiques redoublent dans les années 1750, quand la découverte des peintures d'Herculanum provoque une véritable « querelle de Pline », équivalent artistique de la “ querelle d'Homère » (1715). La gloire d'Apelle et de Zeuxis ne résiste pas à la confrontation avec les médiocres réalisations d'Herculanum. Cette fois, Cochin mène une cabale visant à détruire l'autorité de Pline et le prestige de la peinture ancienne, cabale qui trouve un singulier écho en 1766 dans le débat épistolaire opposant Diderot et Falconet. Dans son commentaire de Pline publié en 1772, Falconet traite Pline de " petit radoteur » et lui reproche d'avoir « compilé sans choix et sans connaissance » des “ bavardages », des " puérilités », bref des récits merveilleux, qui, de tout temps, ont toujours fait l'admiration de la populace. À l'autorité du texte, le sculpteur oppose les leçons de l'expérience, et au modèle idéal, la confrontation directe avec la nature. Parallèlement, Pline est aussi considéré comme une source majeure pour l'histoire de l'art et l'archéologie, avec Caylus, puis Winckelmann et Quatremère de Quincy ; son texte est « rationalisé » et soumis à un examen philologique précis. Cette perspective historique concerne aussi l'iconographie, car la fortune des anecdotes varie selon les époques : la vogue des Apelle et Campaspe est clairement liée à la présence d'un mécénat royal ou princier et atteint son apogée au xvIII siècle. La fortune de Dibutade culmine avec l'exaltation néoclassique de la ligne et la rêverie sur les origines, avant de revenir à la fin du xx siècle comme illustration de la création féminine, dans le sillage des gender studies.

\section{Présentation du site}

L'architecture du site, très simple, apparaît sur la page d'accueil. Elle comprend :

64 * Fortune de Pline. 
1. Un corpus de textes, classés par topos et par ordre alphabétique.

2. Une bibliographie générale des sources, citées en abrégé à l'intérieur des fiches ; ainsi qu'une bibliographie des études critiques.

3. Une rubrique iconographique : le corpus d'images, classées également par topos, avec un lien rattachant l'image à la fiche correspondante.

4. Les rubriques “ Projet » et “ Membres » décrivent les enjeux du projet et mentionnent ses participants.

5. La fonction « recherche » permet de trouver les textes d'un auteur précis, ou n'importe quel terme à l'intérieur des textes ou des traductions (“ sous-textes »). Cette recherche par mot (à condition toutefois de le traduire dans la langue souhaitée) permet de regrouper les textes autour d'une notion, faisant apparaître le rôle théorique des anecdotes.

Au début de chaque fiche figurent l'iconographie et à la bibliographie critique spécifiques au thème. Ensuite, les textes sont déroulés par ordre chronologique, en langue originale (grec, latin, italien, espagnol, portugais, français, anglais, allemand, néerlandais), et si possible avec leurs traductions : pour les textes antiques, une traduction récente est systématiquement donnée ; pour les textes de Pline, fondateurs, sont répertoriées une série de traductions italiennes et

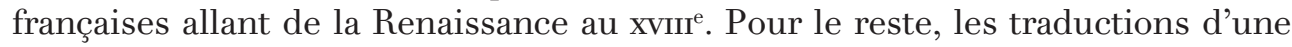
langue à l'autre sont aussi souvent saisies que possible, car elles constituent autant de témoignages de la fortune et de l'interprétation des textes. Les commentaires, en revanche, figurent à la date de leur rédaction. Enfin, les textes sont annotés de façon différenciée : les notes de l'auteur du texte sont réparties en trois catégories (notes en bas de page, notes marginales de référence, placée à droite, ou à fonction de marque-page, placée à gauche), et les notes éditoriales sont limitées au minimum : élucidation d'un terme sous-entendu, ou du contexte quand ce dernier est indispensable à la compréhension.

En conclusion, la dimension collaborative paraît essentielle à un tel projet. Le corpus étant virtuellement infini, la base est appelée à s'enrichir des contributions de chacun. Historiens ou historiens de l'art, philosophes, littéraires, tous sont amenés à rencontrer ces topoi au fil des textes les plus insolites. Souhaitons donc une longue vie à la base Pictor In Fabula.

\author{
Emmanuelle Hénin \\ Université de Reims/CRIMEL \\ 2 , rue de Compiègne \\ 75010 Paris \\ henin.emmanuelle@gmail.com
}

